\title{
Multicast Communications in Multi-Hop Cognitive Radio Networks
}

\author{
Cunhao Gao, Student Member, IEEE, Yi Shi, Member, IEEE, Y. Thomas Hou, Senior Member, IEEE, \\ Hanif D. Sherali, and Huaibei Zhou
}

\begin{abstract}
We study a multicast communication problem in a multi-hop ad hoc network where each node is equipped with a cognitive radio (CR). The goal is to minimize the required network-wide resource to support a set of multicast sessions, with a given bit rate requirement for each multicast session. The unique characteristics and complexity associated with CR distinguish this problem from existing multicast problems for ad hoc networks. In this paper, we formulate this problem via a cross-layer approach by taking consideration of scheduling and routing jointly. Although the problem formulation is in the form of a mixed-integer linear program, we develop a polynomial-time algorithm that offers highly competitive solutions. By comparing the solution values with a lower bound, we show that the proposed algorithm can provide a solution that is close to the optimum.
\end{abstract}

Index Terms-Multicast, resource allocation, cognitive radio, ad hoc networks, optimization.

\section{INTRODUCTION}

$\mathbf{S}$ INCE its inception, cognitive radio (CR) has quickly been accepted as an enabling technology for next-generation wireless communications [1], [2]. Due to its spectrum sensing, learning, and adaptation capabilities, CR is able to address the heart of the problem associated with spectrum scarcity and interoperability. In addition to the well known primary/secondary network setting, cognitive radio has many other important applications [2], [3]. For example, cognitive radio is the key technology for radio interoperability in the US military (i.e., JTRS program [4]), public safety (i.e. SAFECOM program [5]) and future mobile base stations (e.g., see product line from Vanu Inc. [6]). Recently, CR has also been considered as the radio platform for multi-hop ad hoc networks (see, e.g. [7], [8]).

Manuscript received 30 November 2009; revised 10 May 2010 and 14 July 2010. The work of Y.T. Hou and Y. Shi was supported in part by NSF Grant CNS-0721570. The work of H.D. Sherali was supported in part by NSF Grants CMMI-0969169 and ECCS-0925719. The work of H. Zhou was supported by the National Basic Research Program of China (973 Program) under grant 2009CB3204001.

C. Gao is currently with the Department of Electrical and Computer Engineering, Stony Brook University, Stony Brook, NY 11794, USA (e-mail: cgao@ece.sunysb.edu). This work was performed while he was with Virginia Tech.

Y. Shi and Y. T. Hou are with the Bradley Department of Electrical and Computer Engineering, Virginia Tech, Blacksburg, VA 24061, USA (e-mail: \{yshi,thou\}@vt.edu).

H. D. Sherali is with the Grado Department of Industrial and Systems Engineering, Virginia Polytechnic Institute and State University, Blacksburg, VA 24061, USA (e-mail: hanifs@vt.edu).

H. Zhou is with International School of Software Engineering, Wuhan University, China (e-mail: bzhou@whu.edu.cn).

Digital Object Identifier 10.1109/JSAC.2011.110410.
An important service that should be supported by ad hoc networks is multicast. Although there is an abundance of research on multicast in ad hoc networks (see Section II), these results cannot be applied to a CR ad hoc network due to the complexity associated with a CR node (e.g., difference in available bands from neighboring nodes). In addition, a singlelayer approach that is solely focused on multicast connectivity (e.g., multicast routing [9]) is overly simplistic as it does not optimize network resource. In this context, a cross-layer approach is usually necessary, which should include joint consideration of lower layers, in addition to the network layer. However, such a joint formulation is usually highly complex and difficult, as we shall see in this paper (Section IV).

In this work, we consider a multi-hop CR ad hoc network and study how to minimize network-wide resource requirements to support a set of multicast communication sessions. For each multicast session, there is a source node and a group of destination nodes. Further, there is a given bit rate requirement that must be supported from a source node to its group of destination nodes. To minimize required resources to support these multicast communication sessions, we find that it is necessary to follow a cross-layer approach, with joint formulation of frequency band scheduling and multicast routing. Through mathematical modeling, we formulate the optimization problem as a mixed-integer linear program (MILP).

The main contribution of this paper is the development of a polynomial-time algorithm to the cross-layer optimization problem that offers a highly competitive solution. We observe that although there are many integer variables in the problem formulation, the binary scheduling variables (for band assignment on each link) are most significant and their determination helps resolve other integer variables. Based on this observation, we focus on fixing these scheduling variables through an iterative process via a linear programming (LP) relaxation of the original problem. Further, during each iteration, we take an explicit topological consideration when fixing these scheduling variables and follow a "bottom-up" approach (from leaf to root) for multicast tree construction. Once all these integer variables are fixed, the values for the other variables can be obtained by solving an LP.

To measure the performance of our proposed algorithm, we compare its solution to a lower bound obtained via an optimization solver (CPLEX [10]). Note that the optimal solution (unknown) is between the solution obtained by our algorithm and the lower bound by CPLEX. Simulation results show that the solution obtained by our algorithm is very close 
to the lower bound, thus suggesting that the solution obtained by our algorithm is even closer to the optimum. Although the solution in this paper is centralized, it can serve as a performance benchmark for any distributed solution to this problem. A distributed solution is not offered in this paper, which can be studied separately.

The remainder of this paper is organized as follows. Section II reviews related work. In Section III, we study the characteristics associated with multicast communications in a multi-hop CRN, thus laying the ground for mathematical modeling. In Section IV, we present a mathematical model for our problem. In Section $\mathrm{V}$, we present the proposed solution approach for our optimization problem. Section VI presents simulation results and demonstrates the near-optimal performance of our algorithm. Section VII concludes this paper.

\section{RELATED WORK}

In this section, we review related work on multicast in multi-hop ad hoc networks. We organize this section as follows. First, we examine prior efforts on multicast for ad hoc networks with each node equipped with a single traditional radio. Then we review related work on multicast for multichannel multi-radio (MC-MR) ad hoc networks. Although multicast has been studied for single-hop CRNs [11], to the best of our knowledge, there is so far no result on multicast for a multi-hop CRN.

There has been a large body of work on multicast in ad hoc networks with each node equipped with a single traditional (hardware-based) radio, e.g., [12], [13], [14], [15], [16], [17], [18], [19], [20]. Among these works, the focus in [13], [18], [19], [20] was on energy-efficient multicast routing, while the focus in [14], [15], [17] was on lifetime-optimal multicast, either with directional antennas or omni-directional antennas. In [12], [16], the authors studied a multicast throughput maximization problem.

In the context of MC-MR network, multicast was studied in [21], [22]. In [21], Zeng et al. studied a joint routing and channel assignment problem for MC-MR wireless mesh networks with the goal of maximizing throughput. The authors proposed a layer-by-layer decoupled heuristic algorithm called multi-channel multicast (MCM). The MCM algorithm builds a multicast tree via two steps. First, it uses a breadth-firstsearch (BFS)-based algorithm to obtain level information for each node; then it constructs a tree from the lowest level and follows a greedy algorithm in selecting parent nodes. Once the multicast tree is obtained, the channel assignment part commences, where the authors proposed two algorithms, depending on whether the channels are orthogonal or overlapping. In [22], the authors studied channel assignment for a multicast problem in MC-MR wireless mesh networks. Here, the authors first assumed that there is a pre-built multicast tree (e.g., via multicast ad-hoc on-demand distance vector (MAODV) protocol). Then they proposed a channel assignment algorithm that greedily assigns a channel to each transmitting node so as to minimize the interference among nodes in the multicast tree. However, these algorithms for MC-MR networks cannot be applied to address the multicast problem in multi-hop CR

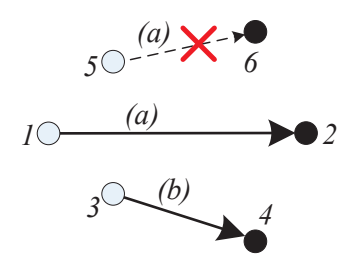

Fig. 1. Additional bands help mitigate interferences in the network and enlarge scheduling space.

networks because MC-MR assumes that there is a set of common channels available for every node in the network, which is hardly true for a multihop CRN.

\section{Understanding the Multicast Problem in A CRN}

In this section, we explore some characteristics associated with the multicast problem in a CRN. Such characteristics are not only important to understand the underlying complexity of our problem, but also help explain our mathematical modeling and problem formulation in Section IV.

Before we describe each characteristic in detail, recall the general setting for an ad hoc CRN. The network that we consider is an ad hoc network with each node equipped with a CR. There is a set of available frequency bands for communication at each node, depending on its location. Such bands may include those bands that are currently not in use by primary users. Given the difference in geographical location, the set of available frequency bands at one node may not be the same as those at another node. In the rest of this section, we discuss some unique and interesting characteristics associated with the multicast problem in a CRN.

Transmission and Interference. Under a traditional wireless network, nodes typically share the same pool of frequency bands. Whether or not two nodes can communicate with each other is only limited by a transmission range. But in a CRN, there is an additional constraint that must be considered. That is, we must make sure that there is at least a common band that is available between the two nodes. If no such band is available, then the two nodes will not be able to communicate with each other.

Under a traditional wireless network, whether or not a node can interfere with another node is only determined by an interference range. Again, such an interference relationship is based on the assumption that all nodes in the network operate on the same frequency band. But for a CRN, the interference relationship is much more complicated due to the potential difference in the set of available frequency bands at different nodes. As an example, Fig. 1 shows three pairs of nodes. Node 1 transmits to node 2 on band $a$ while node 3 transmits to node 4 on band $b$. There is no interference on node 2 because node 3 's transmission is on a different band. But node 5 is not allowed to transmit to node 6 on band $a$ due to its potential interference with node 2.

Multicast Band Scheduling. Under a traditional ad hoc wireless network, a transmission at a node can be heard by all its one-hop neighboring nodes. This is true since all nodes are assumed to operate on the same band. As discussed, this may not be true in a CRN as the available bands on each 


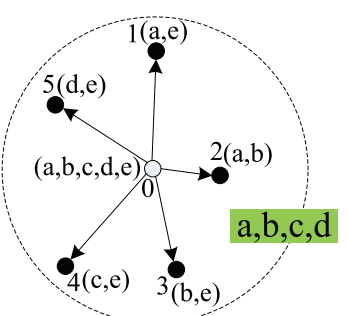

(a) Node 0 uses four bands $\{a, b, c, d\}$ to cover all five neighboring nodes.

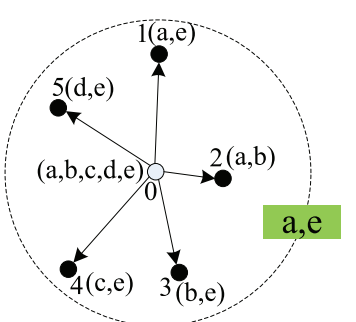

(b) Node 0 uses two bands $\{a, e\}$ to cover all five neighboring nodes.

Fig. 2. Design space for scheduling bands for one-hop multicast.

node may be different and two nodes can communicate with each other only if they share a common band. Due to such diversity in available bands between a source node and its one-hop neighboring nodes, it may be necessary to employ multiple bands to achieve one-hop multicast. As an example, in Fig. 2, suppose that we have a source node 0 with available bands $\{a, b, c, d, e\}$, with node 0 's one-hop neighboring nodes being $1,2,3,4$, and 5 . Assume that the available bands at these five nodes are $\{a, e\},\{a, b\},\{b, e\},\{c, e\}$, and $\{d, e\}$, respectively. It is not difficult to see that there does not exist a common band that node 0 can use to multicast to all its five neighboring nodes. So multiple bands must be used at node 0 to achieve one-hop multicast.

Figures 2(a) and (b) show two possible realizations for this objective. In Fig. 2(a), node 0 uses four bands $\{a, b, c, d\}$ to cover all five nodes; in Fig. 2(b), node 0 uses only two bands $\{a, e\}$ for the same purpose. Given such a difference in band usage, there exists a design (or optimization) space to decide which set of bands to use. The problem is further complicated by the fact that finding the minimum set of bands to cover onehop neighboring nodes does not mean that the total networkwide usage of frequency bands will be minimized, which is our optimization objective (see Section IV-D). This is because a multicast tree will consist of multiple hops and there is interdependency in terms of band usage and interference within the same tree and among different trees.

One-Hop Multicast Rate. A nice property associated with a wireless network is the so-called wireless multicast advantage (WMA) [20], which refers to the phenomenon that the transmission at a node can be heard by all of its one-hop neighboring nodes. But the distances between the transmitting node and its various one-hop neighboring nodes may be different. Given such a difference, the received signal strength at each of the one-hop neighboring nodes will be different, leading to different link rates. For a one-hop multicast, the achievable rate of this hop is therefore limited by the node that is farthest away from the transmitting node.

\section{Modeling, AnAlysis, And Formulation}

We consider a CRN consisting of a set of nodes $\mathcal{V}$ that follows the interweave paradigm [23]. That is, each node $i \in \mathcal{V}$ employs some spectrum sensing techniques (e.g., [24], [25], [26], [27], [28]) to identify a set of available frequency bands $\mathcal{B}_{i}$ that can be used for communications. As discussed, $\mathcal{B}_{i}$ may represent the set of bands that are unused by primary users and may be different at each node due to geographical differences. Denote the set of commonly available bands

TABLE I

NOTATION

\begin{tabular}{|c|c|}
\hline Symbol & Definition \\
\hline $\mathcal{B}_{i}$ & The set of available bands at a node $i \in \mathcal{V}$ \\
\hline $\mathcal{B}$ & $\begin{array}{l}\text { The union of available bands among all the nodes in the } \\
\text { network, } \mathcal{B}=\bigcup_{i \in \mathcal{V}} \mathcal{B}_{i}\end{array}$ \\
\hline $\mathcal{B}_{i j}$ & The set of available bands on link $i \rightarrow j, \mathcal{B}_{i j}=\mathcal{B}_{i} \bigcap \mathcal{B}_{j}$ \\
\hline$c_{i j}^{m}$ & The unicast rate from $i$ to $j$ on band $m$ \\
\hline$c_{i}^{m}$ & The one-hop multicast rate at node $i$ on band $m$ \\
\hline $\mathcal{D}(l)$ & The set of destination nodes of a session $l \in \mathcal{L}$ \\
\hline$d_{i j}$ & Distance between nodes $i$ and $j$ \\
\hline$e_{i j}(l)$ & $\begin{array}{l}\text { Binary indicator to mark whether or not link } i \rightarrow j \text { is used } \\
\text { for session } l\end{array}$ \\
\hline$g_{i j}$ & Path attenuation loss from node $i$ to node $j$ \\
\hline $\mathcal{I}_{j}^{m}$ & $\begin{array}{l}\text { The set of nodes that can have interference at node } j \text { on } \\
\text { band } m\end{array}$ \\
\hline $\mathcal{L}$ & The set of multicast sessions in the network \\
\hline$P$ & The transmission power at a transmitter \\
\hline$R_{T}, R_{I}$ & The transmission and interference ranges \\
\hline$r(l)$ & Multicast flow rate of session $l$ \\
\hline$s(l)$ & Source node of multicast session $l$ \\
\hline $\mathcal{T}_{i}^{m}$ & $\begin{array}{l}\text { The set of nodes that node } i \text { can transmit to and receive } \\
\text { from on band } m\end{array}$ \\
\hline $\mathcal{T}_{i}$ & $\begin{array}{l}\text { The set of nodes that node } i \text { can communicate with on at } \\
\text { least one of its bands, } \mathcal{T}_{i}=\bigcup_{m \in \mathcal{B}_{i}} \mathcal{T}_{i}^{m}\end{array}$ \\
\hline$u_{i j}^{m}(l)$ & $\begin{array}{l}\text { Binary indicator to mark whether or not band } m \text { is assigned } \\
\text { to link } i \rightarrow j \text { for session } l\end{array}$ \\
\hline $\mathcal{V}$ & The set of nodes in the network \\
\hline$W$ & Bandwidth of a frequency band \\
\hline$x_{i}^{m}(l)$ & $\begin{array}{l}\text { Binary indicator to mark whether or not band } m \text { is used for } \\
\text { transmission at node } i \text { for session } l\end{array}$ \\
\hline$y_{j}(l)$ & The depth of node $j$ in the multicast tree for session $l$ \\
\hline$\eta$ & Ambient Gaussian noise density \\
\hline
\end{tabular}

between nodes $i$ and $j$ as $\mathcal{B}_{i j} \equiv \mathcal{B}_{i} \cap \mathcal{B}_{j}$. Denote $\mathcal{L}$ as the set of multicast sessions in the network, with each multicast session consisting of a source node and multiple destination nodes. For a multicast session $l \in \mathcal{L}$, denote $s(l)$ as the source node, $\mathcal{D}(l)$ as the set of destination nodes, and $r(l)$ as a given rate requirement (in $\mathrm{b} / \mathrm{s}$ ) for session $l$. For each multicast session, we consider a tree structure for multicast routing. That is, all the nodes involved in the multicast session $l$ form a tree rooted at node $s(l)$ and with all nodes in $\mathcal{D}(l)$ belonging to the tree. Note that a destination node does not have to be a leaf node on the tree: it may be an intermediate node within the tree. Further, a source node and a destination node of session $l$ may also serve as a relay node for some other session. Table I lists all the notation used in this paper.

We organize this section as follows. Section IV-A presents scheduling and interference models. Section IV-B presents modeling for multicast flow routing. Section IV-C shows how to compute achievable rate for one-hop multicast. Section IV-D elaborates the objective of minimizing the total bandwidth requirement and formulates the problem.

\section{A. Interference Modeling and Scheduling}

Scheduling for transmission at each node in the network can be done either in the frequency domain or time domain. In this paper, we consider scheduling in frequency domain in the form of assigning frequency bands (channels). Since a time domain based formulation is similar to that for frequency domain, it can be shown that our approach can be extended to a time domain based formulation. We first define the following 
binary variable for frequency band scheduling:

$u_{i j}^{m}(l)= \begin{cases}1 & \text { if band } m \text { is used on link } i \rightarrow j \text { for session } l, \\ 0 & \text { otherwise, }\end{cases}$ where $l \in \mathcal{L}, i \in \mathcal{V}, m \in \mathcal{B}_{i}$, and $j \in \mathcal{T}_{i}^{m}, j \neq s(l)$. Here $\mathcal{T}_{i}^{m}$ is the set of nodes to which node $i$ can transmit on band $m$. As we have discussed in Section III, this set includes nodes that are within the transmission range $R_{T}$ of node $i$ and share the same available band $m$, i.e., $\mathcal{T}_{i}^{m}=\{j: j \in \mathcal{V}, 0<$ $\left.d_{i j} \leq R_{T}, m \in \mathcal{B}_{j}\right\}$, where $d_{i j}$ is the distance between nodes $i$ and $j$.

Assuming the granularity of frequency bands can be made small enough for scheduling, we will assign an available band at each transmitting node to at most one multicast session $l \in \mathcal{L}$. That is, if $u_{i j}^{m}(l)=1$ then $u_{i q}^{m}(k)=0$ for another session $k$. This scheduling relationship can be written more compactly as

$$
u_{i q}^{m}(k) \leq 1-u_{i j}^{m}(l),
$$

where $l \in \mathcal{L}, i \in \mathcal{V}, m \in \mathcal{B}_{i}, j \in \mathcal{T}_{i}^{m}, j \neq s(l), k \in \mathcal{L}, k \neq l$, and $q \in \mathcal{T}_{i}^{m}, q \neq s(k)$.

As we have discussed in Section III, when node $j$ is receiving from node $i$ on band $m$, the set of nodes that can interfere with node $j$ should keep silent on band $m$. This set $\mathcal{I}_{j}^{m}$ includes nodes that are within the interference range $R_{I}$ of node $j$ and have the same available band $m$, i.e., $\mathcal{I}_{j}^{m}=\left\{p: p \in \mathcal{V}, d_{p j} \leq R_{I}, m \in \mathcal{B}_{p}\right\}$. Then the scheduling relationship discussed in Section III means $u_{i j}^{m}(l)=1$ will lead to $u_{p q}^{m}(k)=0$ for another link $p \rightarrow q$. This can be written more compactly as

$$
u_{p q}^{m}(k) \leq 1-u_{i j}^{m}(l),
$$

where $l \in \mathcal{L}, i \in \mathcal{V}, m \in \mathcal{B}_{i}, j \in \mathcal{T}_{i}^{m}, j \neq s(l), k \in$ $\mathcal{L}, p \in \mathcal{I}_{j}^{m}, p \neq i$, and $q \in \mathcal{T}_{p}^{m}, q \neq s(k)$. Note that the so-called "self-interference" constraint, i.e., a node cannot transmit and receive on the same band, is embedded in the above constraint (by setting $p=j$ ). Furthermore, by setting $q=j$, the constraint prohibits the same receiving node from simultaneously receiving from two different nodes on the same band (collision), which is consistent with our scheduling in the frequency domain.

\section{B. Multicast Routing}

Multicast Tree Construction. For a multicast session $l \in \mathcal{L}$, its source node $s(l)$ must send its data with a rate $r(l)$ to a set of destination nodes $\mathcal{D}(l)$. We start our mathematical modeling with the following definition:

$$
e_{i j}(l)=\left\{\begin{array}{l}
1 \quad \begin{array}{l}
\text { if link } i \rightarrow j \text { is in the multicast tree for } \\
\text { session } l,
\end{array} \\
0 \quad \begin{array}{l}
\text { otherwise, }
\end{array}
\end{array}\right.
$$

where $l \in \mathcal{L}, i \in \mathcal{V}, j \in \mathcal{T}_{i}, j \neq s(l)$, and $\mathcal{T}_{i}$ denotes the nodes with which node $i$ can communicate on at least one of its bands, i.e., $\mathcal{T}_{i}=\bigcup_{m \in \mathcal{B}_{i}} \mathcal{T}_{i}^{m}$. Note that if $i \in \mathcal{D}(l)$ (i.e., a destination node) and is not a leaf of the tree, then this destination node is an internal node of the tree and helps relay traffic to other destination nodes.

We first formulate the relationship between $e_{i j}(l)$ and $u_{i j}^{m}(l)$. If $e_{i j}(l)=0$, i.e., link $i \rightarrow j$ is not used by the

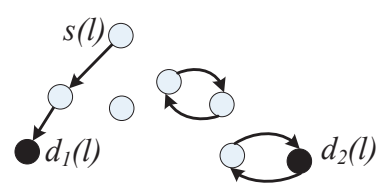

Fig. 3. An example of potential cycle in multicast tree construction.

multicast tree for session $l$, then no band should be assigned to link $i \rightarrow j$ for session $l$. If $e_{i j}(l)=1$, then there must be at least one band assigned to link $i \rightarrow j$ for session $l$. Mathematically, the above relationships can be written as

$$
u_{i j}^{m}(l) \leq e_{i j}(l) \leq \sum_{\rho \in \mathcal{B}_{i j}} u_{i j}^{\rho}(l),
$$

where $l \in \mathcal{L}, i \in \mathcal{V}, j \in \mathcal{T}_{i}, j \neq s(l)$, and $m \in \mathcal{B}_{i j}$.

For a multicast tree for session $l$, the following constraints must be satisfied:

- For a node $i \in \mathcal{V}$, if it is the source node of session $l$, i.e., $i=s(l)$, then it must have at least one out-going edge on the tree. That is,

$$
\sum_{j \in \mathcal{T}_{i}} e_{i j}(l) \geq 1 \quad(l \in \mathcal{L}, i=s(l)) .
$$

- For a node $j \in \mathcal{V}$, if it is a destination node of session $l$, i.e., $j \in \mathcal{D}(l)$, then it must have one incoming edge. That is,

$$
\sum_{i \in \mathcal{T}_{j}} e_{i j}(l)=1 \quad(l \in \mathcal{L}, j \in \mathcal{D}(l)) .
$$

- For a node $j$ that is neither the source node nor a destination node of session $l$, it may be chosen to serve as a relay node in the tree. We have

$$
\sum_{i \in \mathcal{T}_{j}} e_{i j}(l) \leq 1 \quad(l \in \mathcal{L}, j \in \mathcal{V}, j \neq s(l), j \notin \mathcal{D}(l)) .
$$

That is, if node $j$ is a relay node, then there is one edge entering node $j$, otherwise there is no edge entering node $j$. Further, if there is an incoming edge to node $j$, then it must have at least one outgoing edge, i.e., if $\sum_{i \in \mathcal{T}_{j}} e_{i j}(l)=1$, then $\sum_{q \in \mathcal{T}_{j}, q \neq s(l)} e_{j q}(l) \geq 1$; otherwise, no outgoing edge should come out of node $j$, i.e., $\sum_{q \in \mathcal{T}_{j}, q \neq s(l)} e_{j q}(l)=0$. These two scenarios can be mathematically formulated as

$$
\sum_{i \in \mathcal{T}_{j}} e_{i j}(l) \leq \sum_{q \in \mathcal{T}_{j}}^{q \neq s(l)} e_{j q}(l) \leq\left(\left|\mathcal{T}_{j}\right|-1\right) \sum_{i \in \mathcal{T}_{j}} e_{i j}(l)
$$

where $l \in \mathcal{L}, j \in \mathcal{V}, j \neq s(l), j \notin \mathcal{D}(l)$, and $\left(\left|\mathcal{T}_{j}\right|-1\right)$ is an upper bound on the nodes to which $j$ can transmit.

Cycle Prevention. It is important to realize that the constraints described for multicast tree construction still does not exclude the potential existence of cycle(s). As an example, in Fig. 3, source node $s(l)$ attempts to send data to two destinations nodes $d_{1}(l)$ and $d_{2}(l)$. But it is not hard to see that the incorrect solution shown in the figure satisfies all constraints for multicast tree construction. The main reason for this problem arises because when a destination node (or a relay node) receives traffic, there is no mechanism for this 
node to ensure that the traffic it receives is indeed from a node that is one level higher on the multicast tree (i.e., onehop closer to the source node).

To address this problem, we define a new variable $y_{j}(l)$ for each node $j$ to indicate its depth on a multicast tree for session $l$. Specifically, $y_{j}(l)$ is defined as

$$
y_{j}(l)= \begin{cases}-1 & \text { if node } j \text { is not in the tree for session } l, \\ 0 & \text { if node } j \text { is the source node for session } l, \\ h & \text { if node } j \text { has a depth of } h \text { in the tree for } \\ & \text { session } l,\end{cases}
$$

where $l \in \mathcal{L}$ and $j \in \mathcal{V}$. For a node $j$, if it is the source node, then we have

$$
y_{j}(l)=0 \quad(l \in \mathcal{L}, j=s(l)) .
$$

Otherwise, when it is not in the tree, i.e., $\sum_{i \in \mathcal{T}_{i}} e_{i j}(l)=0$, we have $y_{j}(l)=-1$; when it is in the tree, i.e., $\sum_{i \in \mathcal{T}_{j}} e_{i j}(l)=$ 1 , we have $1 \leq y_{j}(l) \leq|\mathcal{V}|-1$. These two cases can be formulated as

$$
2 \sum_{i \in \mathcal{T}_{j}} e_{i j}(l) \leq y_{j}(l)+1 \leq|\mathcal{V}| \sum_{i \in \mathcal{T}_{j}} e_{i j}(l),
$$

where $l \in \mathcal{L}$ and $j \in \mathcal{V}, j \neq s(l)$.

Now we consider the relative depth between two nodes $i$ and $j$. If node $i$ is the parent of node $j$, i.e., $e_{i j}(l)=1$, then $y_{j}(l)-y_{i}(l)=1$. If node $i$ is not the parent of node $j$, i.e., $e_{i j}(l)=0$, then we have $-|\mathcal{V}| \leq y_{j}(l)-y_{i}(l) \leq|\mathcal{V}|$ (due to the fact that both $y_{j}(l)$ and $y_{i}(l)$ are within the interval $[-1,|\mathcal{V}|-1])$. These two cases can be stated mathematically as

$$
\begin{gathered}
y_{j}(l)-y_{i}(l) \geq e_{i j}(l) \cdot(|\mathcal{V}|+1)-|\mathcal{V}|, \\
y_{j}(l)-y_{i}(l) \leq|\mathcal{V}|-(|\mathcal{V}|-1) \cdot e_{i j}(l),
\end{gathered}
$$

where $l \in \mathcal{L}, j \in \mathcal{V}, j \neq s(l)$, and $i \in \mathcal{T}_{j}$.

We now have all the constraints on depth variables $y_{j}(l)$ in (8)-(11). By using these variables, we can ensure a layered structure. The following lemma summarizes our result.

Lemma 1: The use of the $y_{j}(l)$-variables $(j \in \mathcal{V}, l \in \mathcal{L})$ guarantees that the multicast tree is cycle free for each session $l$.

Proof: The proof is based on contradiction. Suppose that constraints (8)-(11) hold and that there is a cycle for session $l$. Assume this cycle consists of $z$ links $i_{1} \rightarrow i_{2}, i_{2} \rightarrow$ $i_{3}, \cdots, i_{z-1} \rightarrow i_{z}$ and $i_{z} \rightarrow i_{1}$, with $z \geq 2$. Then based on (10) and (11), we have $y_{i_{2}}(l)-y_{i_{1}}(l)=1, y_{i_{3}}(l)-y_{i_{2}}(l)=1$, $\cdots, y_{i_{z}}(l)-y_{i_{z-1}}(l)=1, y_{i_{1}}(l)-y_{i_{z}}(l)=1(z \geq 0)$. Summing up all these $z$ equations, all the left-hand side terms cancel out, and the right-hand sides add up to $z$, leading to $0=z$, a contradiction to the assumption that the number of links in the cycle satisfies $z \geq 2$. This completes the proof.

\section{One-Hop Multicast Rate and Flow Rate Constraints}

The formulation for a multicast tree in the last section only addresses our problem from a graph perspective. In this section, we impose additional constraints on the rates in the multicast tree.

One-Hop Multicast Rate. We consider a one-hop multicast where node $i$ transmits to some of its one-hop neighbors on band $m$ simultaneously by taking advantage of wireless multicast advantage (WMA) [20]. Denote $c_{i}^{m}$ as the achievable rate of this one-hop multicast. As discussed in Section III, this rate is limited by the node that is farthest away from node $i$, i.e., $c_{i}^{m}$ is upper bounded by $c_{i j}^{m}=W \log _{2}\left(1+\frac{g_{i j} P}{\eta W}\right)$ for each node $j$ that is receiving on band $m$, where $c_{i j}^{m}$ is the unicast rate to node $j, W$ is the bandwidth of band $m, g_{i j}$ is the path attenuation loss from node $i$ to node $j, P$ is the transmission power, and $\eta$ is the ambient Gaussian noise density. Then for each $i \in \mathcal{V}$ and $m \in \mathcal{B}_{i}$,

$$
c_{i}^{m} \leq c_{i j}^{m} \cdot u_{i j}^{m}(l)+C \cdot\left[1-u_{i j}^{m}(l)\right] \quad\left(j \in \mathcal{T}_{i}^{m}, l \in \mathcal{L}\right),
$$

where $C$ is a sufficiently large number and means that $c_{i}^{m}$ will not be constrained if $u_{i j}^{m}(l)=0$.

In the case that node $i$ is not using band $m$ (i.e., $u_{i j}^{m}(l)=0$ for all $j \in \mathcal{T}_{i}^{m}, l \in \mathcal{L}$ ), then $c_{i}^{m}=0$. This constraint can be written as

$$
c_{i}^{m} \leq C \cdot \sum_{l \in \mathcal{L}} \sum_{j \in \mathcal{T}_{i}^{m}} u_{i j}^{m}(l),
$$

where $c_{i}^{m} \geq 0$ for each $i \in \mathcal{V}$ and $m \in \mathcal{B}_{i}$.

Flow Rate Constraints. Given our modeling for one-hop multicast rate, we now show how they serve as constraints on traffic flows. On each link, we must ensure that the flow rate on this link does not exceed the achievable rate of the link. We have

$r(l) \cdot e_{i j}(l) \leq \sum_{m \in \mathcal{B}_{i j}} u_{i j}^{m}(l) \cdot c_{i}^{m} \quad\left(l \in \mathcal{L}, i \in \mathcal{V}, j \in \mathcal{T}_{i}\right)$,

where $r(l)$ is the constant rate requirement for multicast session $l \in \mathcal{L}$. Note that session $l$ may use multiple bands on link $i \rightarrow j$, which is considered in (14).

To remove the nonlinear term on the right-hand side of (14), we introduce a new variable $f_{i j}^{m}(l)$ to represent $u_{i j}^{m}(l) \cdot c_{i}^{m}$. Then (14) can be rewritten as

$$
r(l) \cdot e_{i j}(l) \leq \sum_{m \in \mathcal{B}_{i j}} f_{i j}^{m}(l) \quad\left(l \in \mathcal{L}, i \in \mathcal{V}, j \in \mathcal{T}_{i}\right) .
$$

Note that $u_{i j}^{m}(l)$ is a binary variable. The nonlinear relationship $f_{i j}^{m}(l)=u_{i j}^{m}(l) \cdot c_{i}^{m}$ can be formulated as

$$
\begin{aligned}
& f_{i j}^{m}(l) \geq 0 \\
& f_{i j}^{m}(l) \leq c_{i}^{m} \\
& f_{i j}^{m}(l) \leq C \cdot u_{i j}^{m}(l) \\
& f_{i j}^{m}(l) \geq C \cdot u_{i j}^{m}(l)-C+c_{i}^{m},
\end{aligned}
$$

where $l \in \mathcal{L}, i \in \mathcal{V}, j \in \mathcal{T}_{i}$ and $m \in \mathcal{B}_{i j}$. Therefore, it is sufficient to have the linear constraints (15)-(19) to represent the nonlinear constraint (14). Such a reformulation is very important as it yields an MILP formulation, instead of a mixed-integer nonlinear program (MINLP). It is well known that an MILP is much easier to handle than a MINLP.

\section{Problem Formulation}

Our objective is to minimize the required network-wide resources to support a set of multicast sessions $\mathcal{L}$ in the network. Resources can be measured in a number of ways. Here, we adopt a new metric called Bandwidth Footprint Product (BFP) to measure the spectral and spatial occupancy of multiband radio communications. BFP was first introduced by Liu 
and Wang (under the name of space-bandwidth product) in [29] and has since been used successfully (see, e.g, [7], [8]) to measure network-wide resource usage in a CRN. Given that we assume the transmission power on each band at each node is $P$, the footprint by each active band is thus identical. Therefore, minimizing network-wide BFP is equivalent to minimizing network-wide bandwidth usage.

To calculate network-wide bandwidth usage, it is necessary to know the set of bands at each node used for transmission. For this purpose, we introduce a binary variable $x_{i}^{m}$, which is defined as

$x_{i}^{m}= \begin{cases}1 & \text { band } m \text { is used at node } i \text { for transmission, } \\ 0 & \text { band } m \text { is not used at node } i \text { for transmission, }\end{cases}$ where $i \in \mathcal{V}$ and $m \in \mathcal{B}_{i}$. Then the objective function is to minimize $\sum_{i \in \mathcal{V}} \sum_{m \in \mathcal{B}_{i}} x_{i}^{m} W$. Since we assume that $W$ is the same for each band, we can drop it from the objective function, i.e.,

$$
\min \sum_{i \in \mathcal{V}} \sum_{m \in \mathcal{B}_{i}} x_{i}^{m} .
$$

Next, consider the constraints on the variables $x_{i}^{m}$. At node $i \in \mathcal{V}$ on band $m \in \mathcal{B}_{i}$, if $u_{i j}^{m}(l)=1$ for any $j \in \mathcal{T}_{i}^{m}$ and $l \in \mathcal{L}$, then $x_{i}^{m}=1$, i.e.,

$$
u_{i j}^{m}(l) \leq x_{i}^{m} \quad\left(\text { for all } j \in \mathcal{T}_{i}^{m} \text { and } l \in \mathcal{L}\right) .
$$

Otherwise, at node $i \in \mathcal{V}$ on band $m \in \mathcal{B}_{i}$, if $u_{i j}^{m}(l)=0$ for all $j \in \mathcal{T}_{i}^{m}$ and $l \in \mathcal{L}$, then $x_{i}^{m}=0$, i.e.,

$$
x_{i}^{m} \leq \sum_{l \in \mathcal{L}} \sum_{j \in \mathcal{T}_{i}^{m}} u_{i j}^{m}(l) .
$$

In summary, our optimization problem is to minimize (20), subject to constraints (1)-(13), (15), (17)-(19), (21), and (22), where $W,|\mathcal{V}|,\left|\mathcal{T}_{j}\right|, \eta, g_{i j}, c_{i j}^{m}, C, P$, and $r(l)$ are constants, while $c_{i}^{m} \geq 0, f_{i j}^{m}(l) \geq 0$ are continuous variables, $y_{j}(l)$ is an integer variable within the interval $[-1,|\mathcal{V}|-1]$, and $u_{i j}^{m}(l)$, $e_{i j}(l)$, and $x_{i}^{m}$ are binary variables. This is a mixed-integer linear program (MILP), which is NP-hard in general [30].

\section{OUR Algorithm}

In this section, we design a polynomial-time algorithm for our optimization problem that offers very competitive solutions. The main mathematical approach employed by the algorithm is to fix a set of integer variables iteratively via an LP relaxation of the original problem. During each iteration, we take explicit topological consideration when fixing integer variables by following a "bottom-up" approach (from leaf to root) for tree construction. In the rest of this section, we give details of these two key ideas in our solution approach and analyze its complexity.

\section{A. Sequential Fixing of u-variables}

For the original MILP, we observe that the $u$-variables have special significance. By definition, $u_{i j}^{m}(l)$ is a binary indicator to mark whether or not band $m$ is assigned to link $i \rightarrow j$ for session $l$. In other words, the variables $u_{i j}^{m}(l)$ are scheduling variables. We find that once the values for $u_{i j}^{m}(l)$ are all determined, the values for $e^{-}, y$ - and $x$-variables can be determined by (3), (9)-(11), (21), and (22), respectively.

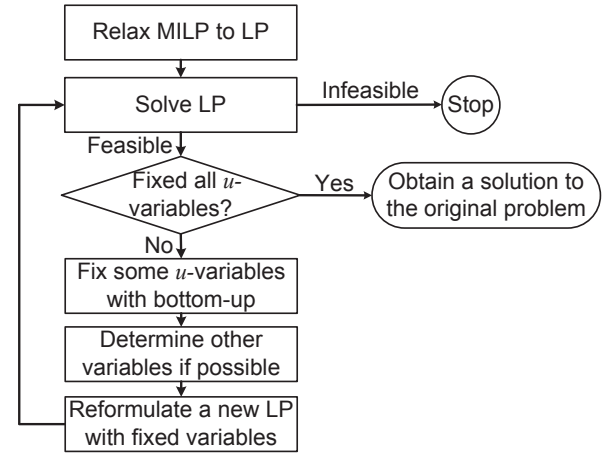

Fig. 4. Flow chart of our proposed solution.

Subsequently, the original MILP can be reduced to an LP, which can be solved in polynomial time. Thus, the key step in solving the MILP is the determination of these binary $u$ variables.

In our proposed solution, we consider a special subset of the $u_{i j}^{m}(l)$-variables at each iteration and fix some of them during the iteration. The choice of such a subset will be explained in detail in Section V-B. Here, we give a basic idea on how the algorithm works.

The flow chart of the proposed solution is shown in Fig. 4. Before the first iteration, we relax all the binary variables. That is, we relax all binary integer variables $u, x$ and $e$ to the interval $[0,1]$ and relax the integer variables $y$ to the interval $[-1,|\mathcal{V}|-1]$. The relaxation of all these integer variables in the original MILP leads to an LP.

After solving this LP, if it is infeasible, then the algorithm stops because there is no feasible solution to support the set of $\mathcal{L}$ multicast sessions in the network. Otherwise, we have a solution to the LP with each $u_{i j}^{m}(l)$ having a value within the interval $[0,1]$. We then consider a special subset of $u$-variables (see Section V-B) and fix one or more of the $u$-variables to 1 based on their closeness to 1 .

Note that the fixing of some $u$-variable to 1 is likely to lead to additional fixing of $x, e, y$, as well as other $u$-variables in the same iteration. For example, suppose $u_{i j}^{m}(l)$ has just been fixed to 1 in this iteration; then we can fix $x_{i}^{m}$ to 1 by (21).

With all the fixed variables in this and past iterations, we can build a new LP and move on to the next iteration. Eventually, all the $u$-variables will be fixed, along with the integer variables $x, e$, and $y$ and a feasible solution to our problem can thus be obtained.

\section{B. Bottom-Up Fixing of Selected u-variables}

We now give details on what special subset of $u$-variables are fixed in each iteration. Recall that our goal is to obtain a multicast tree for each session once all $u$-variables are fixed. If we fix $u$-variables solely based on its closeness to 1 at each iteration (without any joint topological consideration), then we find that the tree construction process is rather chaotic and has the tendency to yield an infeasible solution for a session. As a result, we find that it is most important to incorporate topological considerations when fixing the $u$-variables.

There are two possible approaches to include topological considerations in fixing the $u$-variables. One natural approach is to construct a tree for a session following a "top-down" 


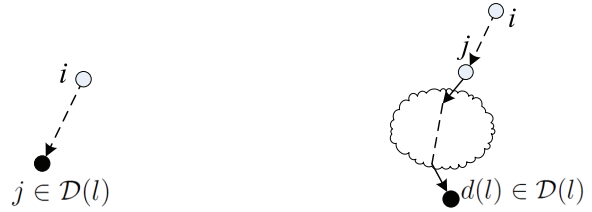

(a) Node $j$ is a destination node (b) Node $j$ already has a route (fixed in of a multicast session $l$. previous iterations) to a destination node of session $l$.

Fig. 5. Selection of subset of $u$ variables to be fixed at each iteration.

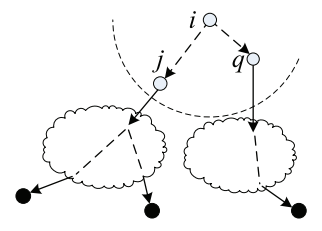

Fig. 6. Merging of two subtrees in bottom-up fixing due to a local broadcast.

approach, i.e., from the root (source node) toward its leaf nodes (destination nodes). The problem with a top-down approach is that when we consider a set of $u$-variables at a node, it is difficult to determine a candidate $u$-variable for the destination nodes. That is, a top-down approach of fixing $u$ variables at a node lacks directional information with respect to the destination nodes. As a result, such an approach suffers from directional "blindness" in fixing the $u$-variables. It is thus not chosen in our approach.

On the other hand, if we start by considering those candidate $u$-variables that are eligible for fixing at the destination nodes and work toward the source node (i.e., "bottom-up" approach), then the direction-blindness problem associated with the topdown approach can be avoided. For this reason, we adopt bottom-up fixing in our solution. Specifically, when we fix $u$-variables, we only consider a subset of those $u_{i j}^{m}(l)$ with either node $j$ being a destination node of a multicast session $l$, or node $j$ having at least one route (which has already been fixed in previous iterations) to a destination node of session $l$ (see Fig. 5). Among all those eligible $u$-variables, we choose the one that is the largest (closest to 1) and fix it to 1 . We call this process a topology-driven fixing algorithm.

Another mechanism that we employ in the bottom-up tree construction is subtree merging. That is, we attempt to exploit WMA as much as possible by joining multiple subtrees with the same node one hop closer to the root. This idea is best illustrated in Fig. 6 where two subtrees with current root nodes $j$ and $q$ are combined together at node $i$ (one hop closer to the source node) with one local broadcast (WMA). However, such joining of multiple sub-trees during multicast construction is performed only when certain criteria are met. In particular, after $u_{i j}^{m}(l)$ is fixed to 1 (i.e., the transmission from node $i$ to node $j$ is performed by a local broadcast), we use the same local broadcast at node $i$ to cover another subtree with root at $q$ (i.e., fix $u_{i q}^{m}(l)$ to 1 ) if (i) $u_{i q}^{m}(l)>0$ in the current relaxed LP solution; and (ii) adding node $q$ into this one-hop multicast group does not increase the total number of required bands at node $i$.

The pseudocode of the proposed algorithm with the bottomup fixing mechanism is given in Fig. 7.

\section{Proposed Algorithm}

1. Set up the initial LP relaxation problem.

2. Solve the LP relaxation. If it is infeasible, then stop and we cannot find a solution to the original problem.

3. If all the $u$-variables are fixed, then stop; and we have a solution to the original problem.

4. Select $u_{i j}^{m}(l)$ that has the largest value among all unfixed $u$-variables with $j$ being either a destination node in $\mathcal{D}(l)$ or has a route to a destination node in $\mathcal{D}(l)$.

5. Find any other node that can be covered by this transmission and fix the corresponding $u$-variables to 1 .

6. Based on constraints (1)-(7), (9)-(11), (21), and (22), fix more $u_{-}, e_{-}, x-$, and $y$-variables.

7. Reformulate the LP relaxation problem with newly fixed variables and go to Step 2.

Fig. 7. Pseudocode of proposed algorithm.

\section{Complexity Analysis}

We now analyze the computational complexity of the proposed algorithm. In particular, we analyze both the number of required iterations in the algorithm and the complexity of each iteration.

To analyze the number of iterations, we note that in each iteration, our algorithm determines an additional link on an available band for a session. Our algorithm derives a solution with a tree topology for routing. For a network with $|\mathcal{V}|$ nodes, a tree has at most $|\mathcal{V}|-1$ edges. Further, each edge within this tree could be active on at most $|\mathcal{B}|$ bands and the number of sessions is $|\mathcal{L}|$. Thus, the number of iterations is no more than the product $(|\mathcal{V}|-1) \cdot|\mathcal{B}| \cdot|\mathcal{L}|$, which is polynomial. Next, we analyze the complexity of each iteration, which involves solving an LP and fixing several variables. There are standard solvers such as CPLEX [10] to solve an LP. The complexity of solving an LP is $O\left(S^{3} L\right)$ [31], where $S$ is the number of variables and $L$ is the number of bits required to store the LP data. The complexity of fixing several variables is much less than solving an LP. Therefore, the complexity of each iteration is $O\left(S^{3} L\right)$. Combining the two results, we conclude that our algorithm has a polynomial-time complexity of $O(|\mathcal{V}| \cdot|\mathcal{B}|$. $\left.|\mathcal{L}| \cdot S^{3} L\right)$.

\section{Simulation Results}

In this section, we present simulation results for our proposed solution. The main goal is to demonstrate the nearoptimal performance of our algorithm.

\section{A. Comparison Metric}

Our algorithm offers a feasible solution to the MILP, which has an objective value (say $F$ ). To study its performance, we should compare $F$ with the optimal objective value (say $O P T)$. Note that the solution obtained by our algorithm cannot be better than the optimal solution, i.e., $\frac{F}{O P T} \geq 1$. If we can show that $\frac{F}{O P T}$ is close to 1 , then our solution is near-optimal. But since an MILP problem is NP-hard in general [30], it cannot be solved via an optimization solver such as CPLEX [10] within reasonable amount of time. Therefore, $O P T$ is unknown and thus $\frac{F}{O P T}$ is also unknown. Nevertheless, we can still implement CPLEX and terminate the computation under a given (tolerable) time limit, e.g., 3 hours, and use the current lower bound obtained by the 
TABLE III

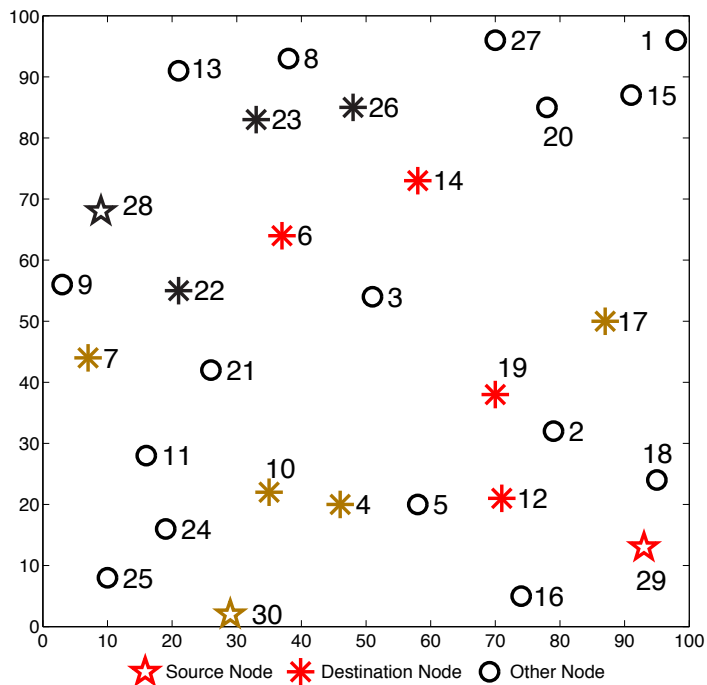

Fig. 8. A 30-node multi-hop CR network used in the example.

TABLE II

SOURCE NODE, DESTINATION NODES, AND RATE REQUIREMENT FOR EACH MULTICAST SESSION IN THE 30-NODE NETWORK EXAMPLE.

\begin{tabular}{|c|c|c|}
\hline Source Node & Destination Nodes & Rate Requirement \\
\hline 29 & $6,12,14,19$ & 76 \\
\hline 30 & $4,7,10,17$ & 94 \\
\hline 28 & $22,23,26$ & 73 \\
\hline
\end{tabular}

solver as a target for comparison. Obviously, the lower bound (say $L B$ ) from CPLEX is less than or equal to $O P T$, i.e., $L B \leq O P T$. Thus, we have $\frac{F}{L B} \geq \frac{F}{O P T} \geq 1$. In this section, we will show that $\frac{F}{L B}$ is close to 1 . As a result, $\frac{F}{O P T}$ is even closer to 1 , i.e., our solution is even closer to the optimum than indicated by $\frac{F}{L B}$.

\section{B. Simulation Setting}

For ease of scalability, we normalize all units for distance, bandwidth, power, and rate with appropriate dimensions. We consider randomly generated ad hoc network topologies consisting of 30 nodes and 40 nodes, respectively. For a 30-node network, the area of deployment is a $100 \times 100$ square whereas for a 40-node network, the area is $125 \times 125$. We assume $|\mathcal{B}|=15$ and $W=50$. The set of available bands at each node is randomly selected from a 15-band pool. The path attenuation loss from node $i$ to node $j$ is $g_{i j}=d_{i j}^{-4}$ and the transmission range and the interference range are set to 30 and 50, respectively. The transmission power $P$ is set to $4 \cdot 10^{7} \eta$.

We assume that there are $|\mathcal{L}|=3$ multicast sessions. For each multicast session, the source node is randomly selected and the set of destination nodes is also randomly selected with a size within $[2,5]$. The rate requirement for each session is randomly chosen within $[40,100]$.

\section{An Example}

Before we present complete simulation results, we examine one instance to gain some insights. The particular instance
EACH NODE'S LOCATION AND AVAILABLE FREQUENCY BANDS FOR THE 30-NODE NETWORK EXAMPLE.

\begin{tabular}{|c|c|c|}
\hline Node & Location & Available Bands \\
\hline 1 & $(98,96)$ & 9,10 \\
\hline 2 & $(79,32)$ & $1,2,4,7,8,9,10,11,13,15$ \\
\hline 3 & $(51,54)$ & $1,2,4,5,6,8,9,10,11,12,13,15$ \\
\hline 4 & $(46,20)$ & $1,3,4,5,7,8,9,10,11,13,14,15$ \\
\hline 5 & $(58,20)$ & $1,2,3,4,5,7,8,9,10,11,13,14,15$ \\
\hline 6 & $(37,64)$ & $1,2,3,4,5,6,7,9,10,12,14$ \\
\hline 7 & $(7,44)$ & $1,3,7,8,9,14$ \\
\hline 8 & $(38,93)$ & $1,2,10$ \\
\hline 9 & $(3,56)$ & $3,6,7,8,9,13,14$ \\
\hline 10 & $(35,22)$ & $1,3,4,5,7,10,11,12,13,14,15$ \\
\hline 11 & $(16,28)$ & $1,3,4,5,7,8,9,12,14$ \\
\hline 12 & $(71,21)$ & $1,2,4,7,8,9,10,11,13,15$ \\
\hline 13 & $(21,91)$ & $3,5,6,10,13,14$ \\
\hline 14 & $(58,73)$ & $1,2,6,9,10,11,12,13$ \\
\hline 15 & $(91,87)$ & $6,9,10,13$ \\
\hline 16 & $(74,5)$ & $2,4,7,9,10,11,13,15$ \\
\hline 17 & $(87,50)$ & $1,2,6,7,8,9,11,13,15$ \\
\hline 18 & $(95,24)$ & $1,2,4,9,15$ \\
\hline 19 & $(70,38)$ & $1,2,4,6,7,8,9,10,11,13,15$ \\
\hline 20 & $(78,85)$ & $2,6,9,10,11,12,13$ \\
\hline 21 & $(26,42)$ & $1,3,4,5,6,7,8,9,10,14$ \\
\hline 22 & $(21,55)$ & $1,3,4,5,6,7,8,9,10,13,14$ \\
\hline 23 & $(33,83)$ & $1,2,3,5,6,10,13,14$ \\
\hline 24 & $(19,16)$ & $1,3,5,7,8,12,14$ \\
\hline 25 & $(10,8)$ & $5,8,12,14$ \\
\hline 26 & $(48,85)$ & $1,2,6,9,10,12$ \\
\hline 27 & $(70,96)$ & $2,9,10,12,13$ \\
\hline 28 & $(9,68)$ & $3,5,6,7,8,9,10,13,14$ \\
\hline 29 & $(93,13)$ & $1,2,4,15$ \\
\hline 30 & $(29,2)$ & $3,5,7,10,12,14$ \\
\hline & & \\
\hline
\end{tabular}

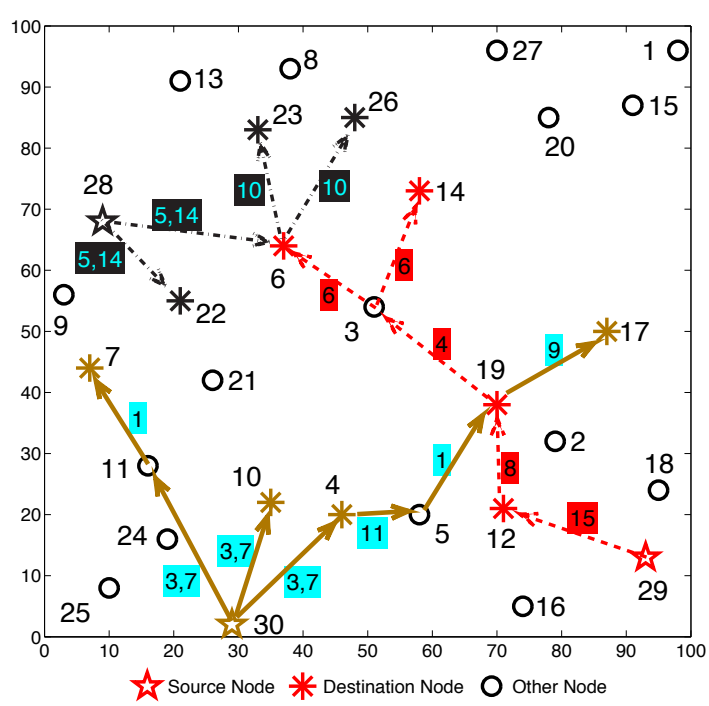

Fig. 9. Solution for the 30-node CRN example.

that we examine is a 30-node network shown in Fig. 8, where pentacles represent source nodes, stars represent destination nodes, and circles represent other nodes in the network. Table II specifies the source nodes, the set of destination nodes for each source, and the rate requirement for each session in the network. The location and available bands of each node are listed in Table III.

The objective value obtained by our solution is 13 , while the lower bound produced by CPLEX is 12 for this instance. 


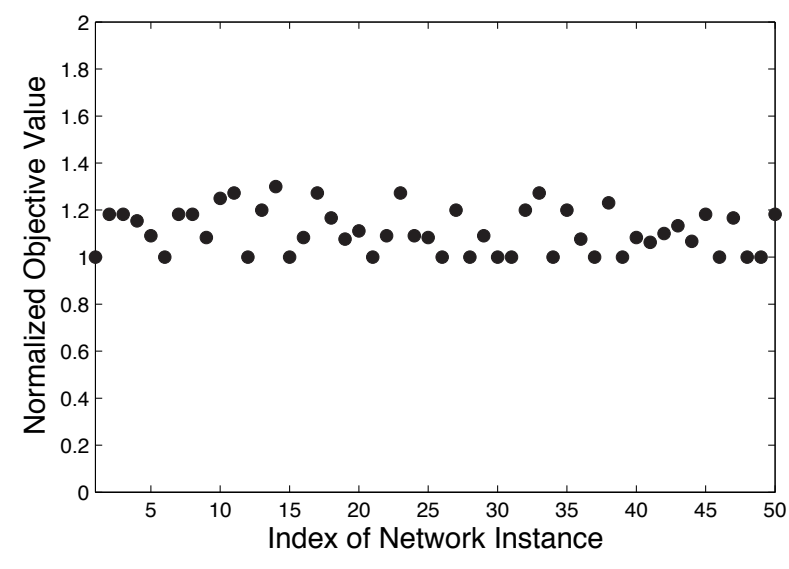

Fig. 10. Results for 50 instances of 30-node multi-hop CR network.

Thus, the normalized result is 1.083 , indicating our solution is very close to the lower bound by CPLEX for this instance.

Fig. 9 displays the details of our results. The numbers embedded in gray and over a link indicate the bands used for this transmission. As shown in the figure, WMA is employed on nodes $3,6,28$, and 30 . Also, note that since session 2 has a high rate requirement (94), one band is not adequate to support this multicast transmission from node 30 to the nodes 4,10 , and 11. As a result, node 30 now uses two bands ( 3 and 7 ) to transmit data to node 4 (also broadcast to nodes 10 and 11).

\section{Simulation Results}

Figs. 10 and 11 present complete results for 30- and 40-node multi-hop CR networks, respectively. For each network size, we randomly generated 50 network instances with different parameter settings (see Section VI-B) and ran both our algorithm and the CPLEX optimization solver (to obtain a lower bound). In each figure, the $x$-axis gives the index for each network instance and the $y$-axis shows the normalized objective value (w.r.t. the lower bound obtained via CPLEX). Therefore, the normalized objective value is always greater than or equal to 1 . Given that the optimal solution must lie between our solution and the lower bound, the closer the normalize objective value to 1 , the better the solution, with 1 being the case that our solution is optimal.

For the 30-node networks, the average normalized objective is 1.111 and the standard deviation is 0.095 and for the $40-$ node networks, the average normalized objective is 1.117 and the standard deviation is 0.082 . Thus, the result from our proposed solution is close to the lower bound obtained by CPLEX. Since the optimal solution is between our result and the lower bound, we conclude that our solution is very close to the optimum and thus is highly competitive. In many cases, the solution by our algorithm coincides with the lower bound, which indicates that our solution is optimal in those cases.

\section{CONCLUSION}

In this paper, we have studied a multicast communication problem in a multi-hop CR network. We showed that the unique characteristics associated with $\mathrm{CR}$ make this problem much more complex and difficult than that for an ad hoc network based on traditional radio. We formulated the resource

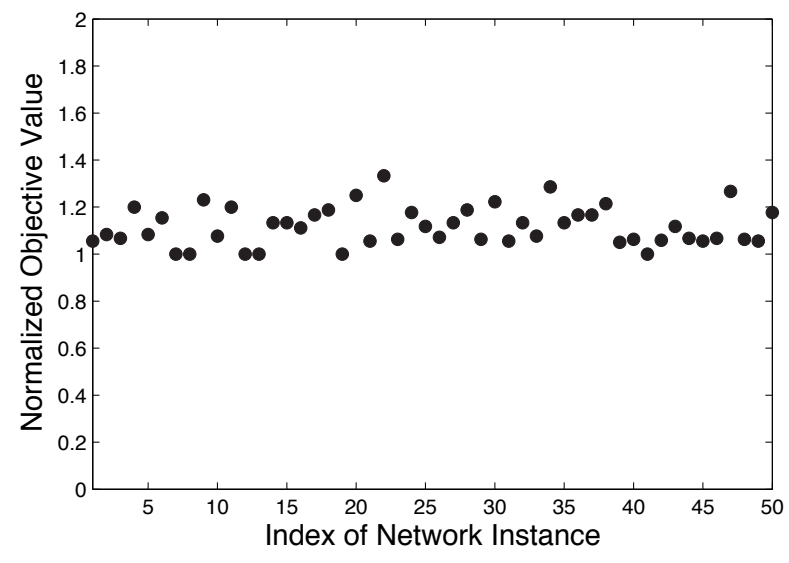

Fig. 11. Results for 50 instances of 40-node multi-hop CR network.

optimization problem as an MILP via a cross-layer approach, and developed a polynomial-time algorithm that incorporates several novel ideas. Based on simulation results, we demonstrated that the proposed algorithm offers competitive solutions. The solution in this paper is centralized and can be used as a performance benchmark for any distributed solution to this problem. A distributed solution will be studied separately in our future work.

\section{REFERENCES}

[1] S. Haykin, "Cognitive radio: Brain-empowered wireless communications," IEEE J. Sel. Areas Commun., vol. 23, no. 2, pp. 201-220, Feb. 2005.

[2] A.M. Wyglinski, M. Nekovee, and Y.T. Hou (eds.), Cognitive Radio Communications and Networks: Principles and Practice, Chapter 1, Academic Press/Elsevier, 2010.

[3] I.F. Akyildiz, W.-Y. Lee, M.C. Vuran, and S. Mohanty, "Next generation/dynamic spectrum access/cognitive radio wireless networks: A survey," Computer Networks, vol. 50, no. 13, pp. 2127-2159, Sept. 2006.

[4] Joint Tactical Radio System, http://www.globalsecurity.org/military/ systems/ground/jtrs.htm.

[5] SAFECOM - http://www.safecomprogram.gov/SAFECOM/.

[6] Vanu Inc., http://www.vanu.com/solutions/solutions.html.

[7] Y.T. Hou, Y. Shi, and H.D. Sherali, "Spectrum sharing for multi-hop networking with cognitive radios," IEEE J. Sel. Areas Commun., vol. 26, no. 1, pp. 146-155, Jan. 2008.

[8] Y. Shi, Y.T. Hou, and H. Zhou, "Per-node based optimal power control for multi-hop cognitive radio networks," IEEE Trans. Wireless Commun., vol. 8, no. 10, pp. 5290-5299, Oct. 2009.

[9] E.M. Royer and C.E. Perkins, "Multicast operation of the ad-hoc ondemand distance vector routing protocol," in Proc. ACM MobiCom, pp. 207-218, Seattle, WA, Aug. 1999.

[10] IBM ILOG CPLEX Optimizer, http://www-01.ibm.com/software/ integration/optimization/cplex-optimizer/.

[11] D. Hu, S. Mao, Y.T. Hou, and J.H. Reed, "Scalable video multicast in cognitive radio networks," IEEE J. Sel. Areas Commun., vol. 28, no. 3, pp. 434-444, April 2010.

[12] R. Bhatia and L. Li, "Characterizing achievable multicast rates in multihop wireless networks," in Proc. ACM MobiHoc, pp. 133-144, UrbanaChampaign, IL, May 2005.

[13] A.K. Das, R.J. Marks, M. El-Sharkawi, P. Arabshahi, and A. Gray, "Minimum power broadcast trees for wireless networks: Integer programming formulations," in Proc. IEEE INFOCOM, pp. 1001-1010, San Francisco, CA, March 2003.

[14] S. Guo, M. Guo, and V. Leung, "Exploring the multicast lifetime capacity of WANETs with directional multibeam antennas," in Proc. IEEE INFOCOM, pp. 2686-2690, Rio de Janeiro, Brazil, April 2009.

[15] Y.T. Hou, Y. Shi, and H.D. Sherali, "Multicast communications in ad hoc network using directional antennas: A lifetime-centric approach," IEEE Trans. Veh. Technol., vol. 56, no. 3, pp. 1333-1344, May 2007.

[16] W. Liang and X. Guo, "Online multicasting for network capacity maximization in energy-constrainted ad hoc networks," IEEE Trans. Mobile Comput., vol. 5, no. 9, pp. 1215-1227, Sept. 2006. 
[17] A. Orda and B.-A. Yassour, "Maximum-lifetime routing algorithms for networks with omnidirectional and directional antennas", in Proc. ACM MobiHoc, pp. 426-437, Urbana-Champaign, IL, May 2005.

[18] P-J. Wan, G. Calinescu, and C-W. Yi, "Minimum-power multicast routing in static ad hoc wireless networks," IEEE/ACM Trans. Netw., vol. 12 , no. 3, pp. 507-514, June 2004.

[19] J.E. Wieselthier, G.D. Nguyen, and A. Ephremides, "Algorithms for energy-efficient multicasting in static ad hoc wireless networks," ACM/Springer Mobile Networks and Applications (MONET), vol. 6, no. 3, pp. 251-263, June 2001.

[20] J.E. Wieselthier, G.D. Nguyen, and A. Ephremides, "On the construction of energy-efficient broadcast and multicast trees in wireless networks," in Proc. IEEE INFOCOM, pp. 585-594, Tel Aviv, Israel, March 2000.

[21] G. Zeng, B. Wang, Y. Ding, L. Xiao, and M. Mutka, "Multicast algorithms for multi-channel wireless mesh networks," in Proc. IEEE Int'l Conf. on Network Protocols (ICNP), pp. 1-10, Beijing, China, Oct. 2007.

[22] H.L. Nguyen and U.T. Nguyen, "Channel assignment for multicast in multi-channel multi-radio wireless mesh networks," Wireless Communications and Mobile Computing, vol. 9, no. 4, pp. 557-571, April 2009.

[23] A. Goldsmith, S.A. Jafar, I. Maric, and S. Srinivasa, "Breaking spectrum gridlock with cognitive radios: An information theoretic perspective," Proc. IEEE, vol. 97, no. 5, pp. 894-914, May 2009.

[24] D. Cabric, S.M. Mishra, and R.W. Brodersen, "Implementation issues in spectrum sensing for cognitive radios," in Proc. IEEE Asilomar Conference on Signals, Systems and Computers, pp. 772-776, Pacific Grove, CA, Nov. 7-10, 2004

[25] G. Ganesan and Y.G. Li, "Cooperative spectrum sensing in cognitive radio networks," in Proc. IEEE DySPAN, pp. 137-143, Baltimore, MD, Nov. 8-11, 2005.

[26] A. Gashemi and E. Sousa, "Collaborative spectrum sensing for opportunistic access in fading environments," in Proc. IEEE DySPAN, pp. 131-136, Baltimore, MD, Nov. 8-11, 2005.

[27] S.M. Mishra, A. Sahai, and R.W. Brodersen, "Cooperative sensing among cognitive radios," in Proc. IEEE International Conference on Communications, pp. 1658-1663, Istanbul, Turkey, June 11-15, 2006.

[28] T. Renk, C. Kloeck, and F.K. Jondral, "A cognitive approach to the detection of spectrum holes in wireless networks," in Proc. IEEE Consumer Communications and Networking Conference, pp. 11181122, Las Vegas, NV, Jan. 11-13, 2007.

[29] X. Liu and W. Wang, "On the characteristics of spectrum-agile communication networks," in Proc. IEEE DySPAN, pp. 214-223, Baltimore, MD, Nov. 2005.

[30] M.R. Garey and D.S. Johnson, Computers and Intractability: A Guide to the Theory of NP-completenes, W.H. Freeman and Company, pp. 245248, New York, NY, 1979.

[31] M.S. Bazaraa, J.J. Jarvis, H.D. Sherali, Linear Programming and Network Flows, Fourth Edition, John Wiley \& Sons, 2010.

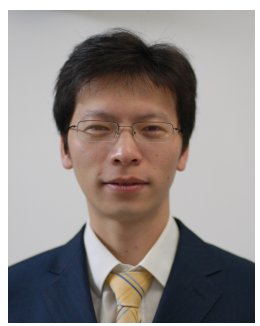

Cunhao Gao (S'08) received his B.S. and M.E. degree in Computer Science from University of Science and Technology of China, Hefei, China, in 2004 and 2007, respectively. In 2009, he earned his M.S. degree in Computer Engineering from the Bradley Department of Electrical and Computer Engineering, Virginia Polytechnic Institute and State University (Virginia Tech), Blacksburg, VA. He is currently working toward his Ph.D. degree in the department of Electrical and Computer Engineering at Stony Brook University, NY. His current research focuses on cross-layer design and optimizations for wireless networks, ad hoc networks, cognitive radio networks, and MIMO.

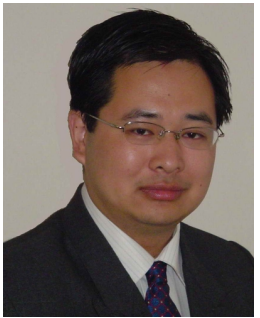

Yi Shi (S'02-M'08) received his Ph.D. degree in computer engineering from Virginia Tech, in 2007. $\mathrm{He}$ is currently a Research Scientist in the Department of Electrical and Computer Engineering at Virginia Tech.

His research focuses on algorithms and optimization for cognitive radio networks, MIMO and cooperative communication networks, sensor networks, and ad hoc networks. He was a recipient of IEEE INFOCOM 2008 Best Paper Award. He was a recipient of Chinese Government Award for Outstanding Ph.D. Students Abroad in 2006. While an undergraduate, he was a recipient of Meritorious Award in International Mathematical Contest in Modeling in 1997 and 1998, respectively. He served as a TPC member for many major international conferences (including ACM MobiHoc 2009 and IEEE INFOCOM 2009-2011).

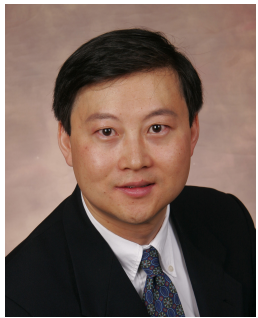

Y. Thomas Hou (S'91-M'98-SM'04) received his $\mathrm{Ph} . \mathrm{D}$. degree in Electrical Engineering from Polytechnic Institute of New York University in 1998. From 1997 to 2002, Dr. Hou was a Researcher at Fujitsu Laboratories of America, Sunnyvale, CA Since 2002, he has been with Virginia Polytechnic Institute and State University ("Virginia Tech"), the Bradley Department of Electrical and Computer Engineering, Blacksburg, VA, where he is now an Associate Professor.

Prof. Hou's research interests are cross-layer design and optimization for cognitive radio wireless networks, cooperative communications, MIMO-based ad hoc networks, and new interference management schemes for wireless networks. He is a recipient of an Office of Naval Research (ONR) Young Investigator Award (2003) and a National Science Foundation (NSF) CAREER Award (2004) for his research on optimizations and algorithm design for wireless ad hoc and sensor networks.

Prof. Hou is currently serving as an Area Editor of IEEE Transactions on Wireless Communications, and Editor for IEEE Transactions on Mobile Computing, IEEE Wireless Communications, ACM/Springer Wireless Networks (WINET), and Elsevier Ad Hoc Networks. He was a past Associate Editor of IEEE Transactions on Vehicular Technology. He was Technical Program Co-Chair of IEEE INFOCOM 2009.

Prof. Hou recently co-edited a textbook titled Cognitive Radio Communications and Networks: Principles and Practices, which was published by Academic Press/Elsevier, 2010.

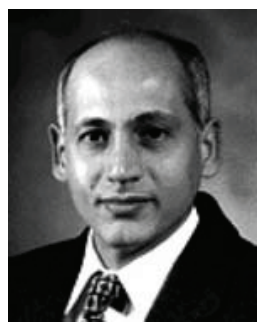

Hanif D. Sherali is a University Distinguished Professor and the W. Thomas Rice Chaired Professor of Engineering in the Industrial and Systems Engineering Department at Virginia Polytechnic Institute and State University. His areas of research interest are in analyzing problems and designing algorithms for specially structured linear, nonlinear, and integer programs arising in various applications, global optimization methods for non-convex programming problems, location and transportation theory and applications, and economic and energy mathematical modeling and analysis. He has published over 271 refereed articles in various Operations Research journals, has (co-) authored seven books in this area, and serves on the editorial board of eight journals. He is an elected member of the National Academy of Engineering.

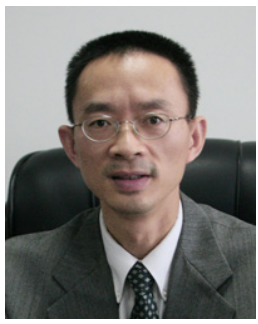

Huaibei (Barry) Zhou received his B.S and M.S degrees in Physics from Wuhan University, China in 1984 and 1987, respectively, and his Ph.D. degree in Physics from Univ. of Maryland, College Park, in 1994. His previous experience includes Postdoctoral Fellow at National Institute of Standard and Technology (NIST) in Maryland (1994-1996), Senior Engineer at GE (1996-1999) and Senior Technical Manager at NEXTEL (1999-2002). He is currently Dean of International School of Software Engineering, Wuhan University, China. His current research interests include cognitive radio and radio wave propagation. 